\title{
Rosen und Disteln. Geburtstagsbriefe an Stefan George
}

\author{
Eine Kabinettausstellung
}

Am 12. Juli 2018 jährte sich Stefan Georges

Geburtstag zum 150. Mal. Die Kabinettausstellung, die aus diesem Anlass vom 28. Juni bis 10. August in der Württembergischen Landesbibliothek zu sehen war, ließ an den Dichter gerichtete Geburtstagsbriefe aus den Jahren 1896 bis 1933 noch einmal sprechen. Der Obertitel der Ausstellung war dabei von George selbst entlehnt: „Rosen und Disteln" hieß die Schülerzeitschrift, die der Gymnasiast gemeinsam mit Schulfreunden 1887 in nur einer Nummer herausgebracht hatte. In der Spannung dieses Titels bewegte sich auch die Ausstellung, indem sie die immer lobenden, gewissermaßen Rosen überbringenden Geburtstagsbriefe zeigte und zugleich die mitunter stachligen Kontexte rekonstruierte.

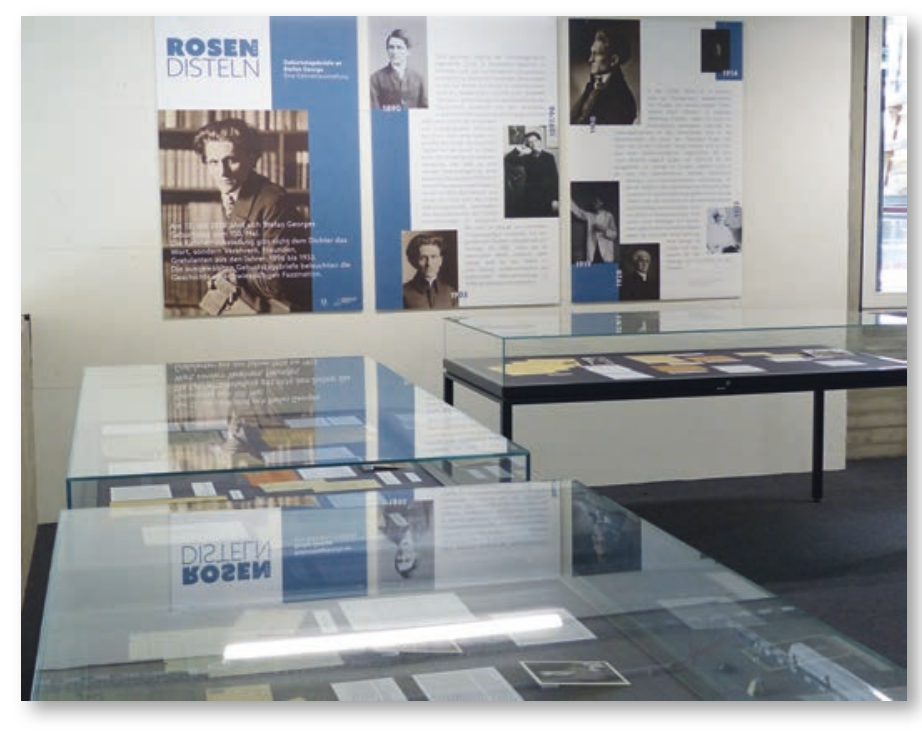

Abb. 1: Blick in die Ausstellung

Aber wer war Stefan George? 1868 geboren, beginnt der sprachbegeisterte Jugendliche schon zu Schulzeiten, Gedichte zu schreiben und aus verschiedenen europäischen Literaturen zu übersetzen. Nach dem Abitur begibt er sich auf Reisen und knüpft quer durch Europa künstlerische Kontakte. "Hymnen“, Stefan Georges erstes Gedichtbuch, das den literarischen Austausch mit den wichtigsten ästhetischen Strömungen seiner Zeit wagt, erscheint 1890. Es spricht der Einsamkeit und homoerotischen Sehnsucht das Wort und tritt mit dem Anspruch an, die deutsche Lyrik erneuern zu wollen. Zwei Jahre später begründet der Dichter die Zeitschrift „Blätter für die Kunst” als Organ eines sich ausweitenden, am Symbolismus orientierten Künstlernetzwerkes. Von 1897 an wird George, bald dreißigjährig, einer breiteren literarischen Öffentlichkeit mit "Das Jahr der Seele" bekannt, seinem fünften Gedichtbuch. Aus dieser Zeit sind auch die ersten Geburtstagsbriefe überliefert.

Schon den Glückwünschen der frühen Künstlerfreunde ist abzulesen, wie sehr sie George zur kulturellen Erneuerungsfigur stilisieren. Die nächsten drei Dekaden spiegeln den Erfolg dieser Idee. Um 1900 bereits fest im literarischen Markt etabliert, steht George bald für den Willen zu einer fundamental modernekritischen Lebensreform in Dichtung, Wissenschaft und Kultur. Ab dieser Zeit versammelt der frühere Dichter der Einsamkeit dichtungsbegeisterte junge Männer, zumeist Studenten und junge Wissenschaftler, aber auch Adoleszente um sich. Der Autor des "Siebenten Rings" (1907), des "Stern des Bundes" (1914) und des "Neuen Reichs" (1928) im Zentrum dieser von der Öffentlichkeit als "George-Kreis" wahrgenommenen Gruppierungen wird zum Idol einer elitär-antimodernen, auf ein vermeintlich besseres "geistiges" Deutschland ausgerichteten Gegenkultur, die eine "neue deutsche Jugend" prägen will. Während die persönlichen Abhängigkeiten um George verschattet bleiben, sind es die Linien von Jugendemphase, Zeitkritik, bündischem Elitismus und nationalkulturellem Aufschwung in seinem Werk, für die der Dichter in den Jubiläumsjahren 1918 und 1928, zu seinem 50. und 60. Geburtstag, gefeiert wird. Dass diese Linien ihn schließlich für die Nationalsozialisten anschlussfähig erscheinen lassen, wird um 1933 zum Lagerstreit unter seinen Anhängern. George selbst, auf Distanz, aber zugleich ohne nachhaltige Abwehr gegen die Vereinnahmungsversuche der Nazis, stirbt ein halbes Jahr 
nach seinem 65. Geburtstag, am 4.12.1933, in der Schweiz.

Auf seinem Lebensweg durch das endende 19. und das beginnende 20. Jahrhundert bekam George Glückwunschpost: Familienmitglieder, frühe Künstlergenossen, dann Freunde aus dem „Kreis", mit zunehmender Berühmtheit offizielle Repräsentanten staatlicher Institutionen, aber auch lyrikbegeisterte Fans, die an seinem Ruhm teilhaben wollten, gratulierten ihm zum Geburtstag.

In vier Großvitrinen präsentierte die Ausstellung eine chronologisch sortierte Auswahl mit Briefen von Anna Ottilie George (1896), Melchior Lechter (1897), Karl Wolfskehl (1898), Willem de Haan (1900), Berthold Vallentin (1904), Friedrich Gundolf (1911), Wilhelm Walther (1911), Karl Bauer (1918), Adalbert Cohrs (1918), Ernst Morwitz (1918), einer unbekannten jungen Mutter (1918), Walter Elze (1926), Reichspräsident Paul von Hindenburg (1928), der Preußischen Akademie der Künste (1928), Kultusminister Carl Heinrich Becker (1928), Edith Landmann (1928), Claus Schenk Graf von Stauffenberg (1931), Ernst Gundolf (1933), Propagandaminister Joseph Goebbels (1933) und Ernst H. Kantorowicz (1933). Aus den zwanzig ausgestellten Exponaten werden hier acht Briefe mit Transkriptionen und den erläuternden Begleittexten wiedergegeben.

\section{Melchior Lechter an Stefan George, 12.7.1897}

Widmung auf Kaschierkarton als Rückseite zu Lechters „Inspiration" von 1893

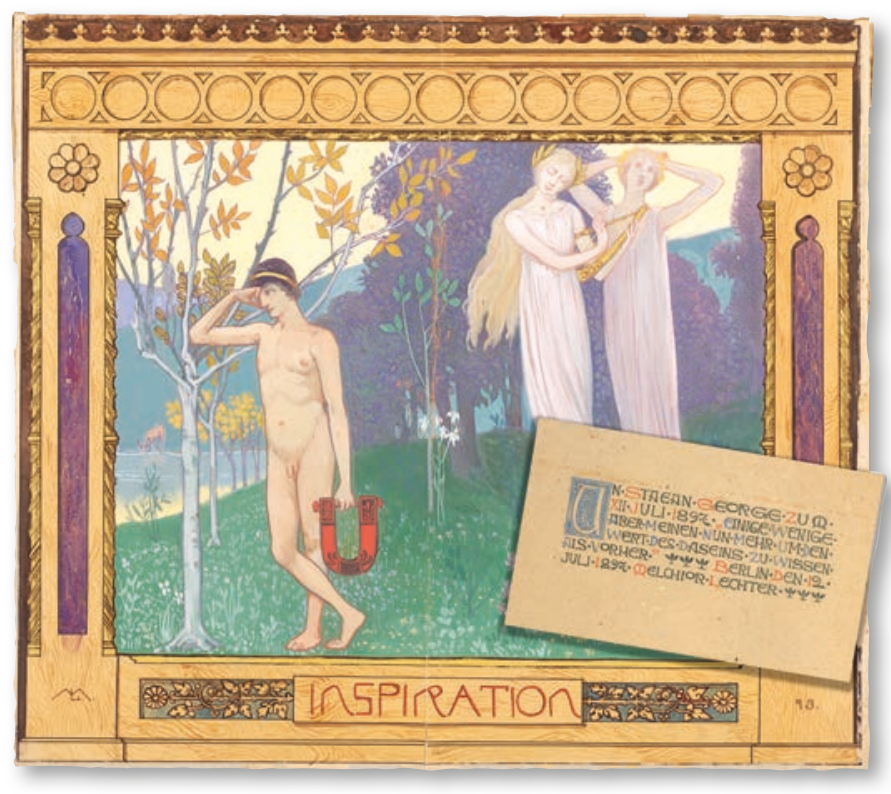

\section{Transkription}

„AN STAEAN ${ }^{1}$ GEORGE ZUM XII JULI 1897 ,EINIGE WENIGE ABER MEINEN NUN MEHR UM DEN WERT DES DASEINS ZU WISSEN ALS VORHER'2 BERLIN DEN 12 JULI 1897 MELCHIOR LECHTER“

\section{Erläuterung}

Der Entwurf des Glasmalers und Buchgestalters Melchior Lechter (1865-1937) zu einem dann nicht ausgeführten Wandgemälde mit dem Titel "Inspiration" (das Pendant "Musenhain" ist im LWLMuseum für Kunst und Kultur in Münster erhalten) entstand bereits 1893 und damit wohl unmittelbar nach dem Erstkontakt Lechters mit Werken Georges. Lechter war im Herbst 1893 über Hugo von Hofmannsthal auf Georges Zeitschrift „Blätter für die Kunst" aufmerksam geworden und kam so in Berührung mit Gedichten Georges. In ihnen sieht er seine eigenen ästhetischen Auffassungen gleichsam mitausgesprochen und sucht daraufhin den Kontakt zu ihrem Autor.

"Inspiration", ein Jugendstilszenario, das die Abwendung eines antikisierten, eine Lyra als Dichtersymbol tragenden nackten Jünglings von den weiblichen Musen und ihrem Gesang bebildert - ein Motiv, das schwebend in Georges früher Lyrik anwesend ist -, schenkt Lechter George zum 29. Geburtstag und verneigt sich in seiner beigefügten Widmung vermittels des Hofmannsthal-Zitats vor ihm. Im selben Jahr, 1897, erscheint mit "Das Jahr der Seele" dann das erste Buch Georges in Gestaltung von Melchior Lechter. Die professionelle Zusammenarbeit der befreundeten Künstler wird gut zehn Jahre andauern. Der sakralisierende, das Gesamtkunstwerk anstrebende, später auch esoterisch durchwirkte Jugendstil Lechters, den George in Abkehr von der strengen Gestaltung seiner ersten Privatdrucke wählt, hat die Wahrnehmung seiner Werke deutlich mitgeprägt. 


\section{Willem de Haan an Stefan George (bei Albert Verwey), 12.7.1900}

Briefkarte, 2 Seiten, mit Umschlag

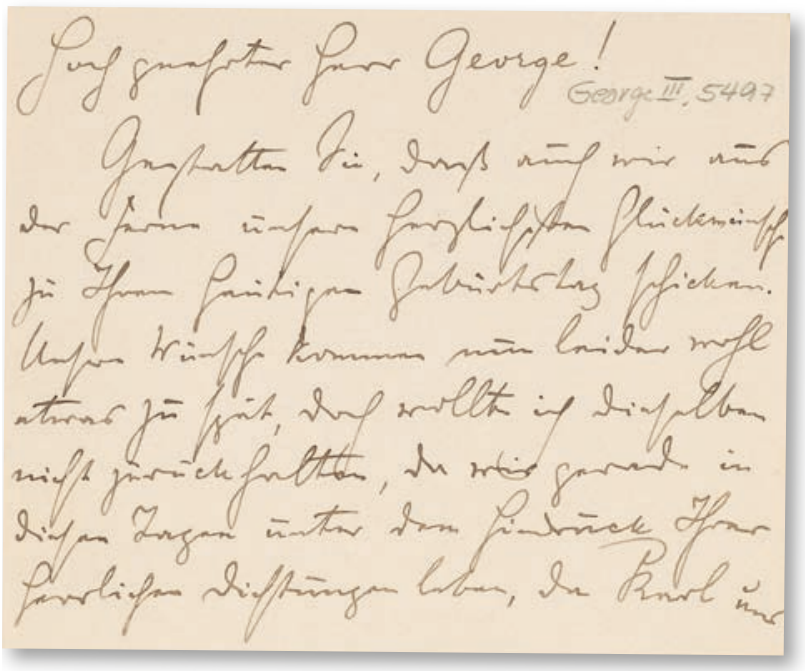

\section{Transkription}

"Hochgeehrter Herr George!

Gestatten Sie, daß auch wir aus der Ferne unsere herzlichsten Glückwünsche zu Ihrem heutigen Geburtstag schicken. Unsere Wünsche kommen nun leider wohl etwas zu spät, doch wollte ich dieselben nicht zurückhalten, da wir gerade in diesen Tagen unter dem Eindruck Ihrer herrlichen Dichtungen leben, da Karl uns vorgestern Abend, in einem kleinen Kreise bei uns, Einiges aus dem Teppich des Lebens zu Aller Entzücken vortrug. Haben Sie tausend Dank für das viele Herrliche, welches Sie uns und der Menschheit geschenkt haben, und möge ein gütiges Geschick Ihnen Kraft und Begeisterung erhalten noch manches Kunstwerk zu schaffen!

Mit den herzlichsten Grüßen von den Meinigen, und mit der Bitte, mich unbekannterweise Herrn Verwey empfehlen zu wollen, zeichne ich in treuer Verehrung

Darmstadt, 12/7 1900. Ihr ergebenster W. de Haan"

\section{Erläuterung}

Der Niederländer Willem de Haan (1849-1930), seit 1881 Hofkapellmeister in Darmstadt und über Jahrzehnte die zentrale Figur im Musikleben der Stadt, war der Schwiegervater von Georges frühem Künstlerfreund Karl Wolfskehl. Auch wenn George in Darmstadt zur Schule gegangen war, fand die erste persönliche Begegnung zwischen beiden wohl erst Ende 1898 bei der Hochzeit von de Haans Tochter Hanna mit Wolfskehl statt. Im darauffolgenden Jahr bereits vertonte Willem de Haan einige Gedichte Georges und besuchte inn zusammen mit seiner Frau in Bingen. "Der Teppich des Lebens" war Georges sechstes Gedichtbuch. Symbolträchtig mit der Jahreszahl 1900 versehen, erschien der großformatige, in grünes Leinen gebundene, messbuchhafte Prachtband Ende 1899 in nur 300 nummerierten Exemplaren in Ausstattung Melchior Lechters. Mit dem auf die Marktmacht der Exklusivität ausgerichteten, pseudosakralen "Wunderwerk" (Wolfskehl) inszenierte George seine Gedichte als Ereignis der Jahrhundertwende und zielte auf breitere Wirkung. Lesungen aus seinen Gedichten, wie sie später ein festes Ritual bei den Zusammentreffen seiner Anhänger bildeten, dienten in diesen frühen Jahren oft auch als Mittel der Werbung.

Willem de Haans abschließender Gruß an den niederländischen Dichter Albert Verwey, bei dem sich George gerade aufhielt und an dessen Adresse in Noordwyk der Brief adressiert ist, verweist auf die engen Verbindungen zu anderen europäischen Symbolisten, die George seit Anfang der 1890er Jahre aufgebaut hatte und unterhielt. Zu denken ist etwa an die Franzosen Stéphane Mallarmé und Albert SaintPaul, den Polen Wacław Rolicz-Lieder, den Belgier Albert Mockel oder eben den Niederländer Verwey.

\section{Friedrich Gundolf an Stefan George, 11.7.1911}

1 Blatt, 2 Seiten

$$
\begin{aligned}
& \text { Tenenster: Gesteen war ith bee II. } \\
& \text { Die Mater bithet dw mógest sellst mis ner } \\
& \text { threm mitkilen waxd der ihn brautew } \\
& \text { koialest. Un Anfary sugust at habe es } \\
& \text { Zit. Wh las gestern dost aus dem DIV. Ring. }
\end{aligned}
$$

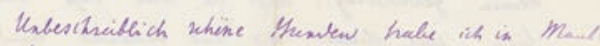

$$
\begin{aligned}
& \text { Imm getrabs und ish verde div kene Ruke } \\
& \text { lopew bis du mit mis dest sarss smajges } \\
& \text { an eveni dillen sommentay um die Tagogeintes: } \\
& \text { there - es ist eni thatle gesammeller undentrobs } \\
& \text { montister traumvelstany, eines Tafel uni DII } \\
& \text { Ring wert bre nur irgead ain dentsches mal. } \\
& \text { auch var ith in dem nakem Knitherijen, vo } \\
& \text { man das quentshaus des doper Faud yijh. }
\end{aligned}
$$

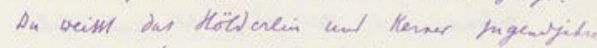

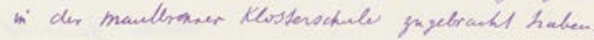

$$
\begin{aligned}
& \text { Enet des lang sheilt mis heute dass er } \\
& \text { milifär untanglish befundew erende : des } \\
& \text { Beilterstern }
\end{aligned}
$$




\section{Transkription}

„Teuerster: Gestern war ich bei SS. Die Mater bittet du mögest selbst mir oder Ihnen mitteilen wann du ihn brauchen könntest. Von Anfang August ab habe er Zeit. Ich las gestern dort aus dem VII. Ring.

Unbeschreiblich schöne Stunden habe ich in Maulbronn gehabt und ich werde dir keine Ruhe lassen bis du mit mir dort warst womöglich an einem stillen sommertag um die Tagsgeisterstunde - es ist eine stätte gesammelter urdeutscher, romantischer traumsubstanz, einer Tafel im VII. Ring wert wie nur irgend ein deutsches Mal. Auch war ich in dem nahen Knittlingen, wo man das Geburtshaus des doktor Faust zeigt. Du weisst das [sic!] Hölderlin und Kerner jugendjahre in der maulbronner Klosterschule zugebracht haben.

Ernst der lange schreibt mir heute dass er Militär untauglich befunden wurde: der Blätterstern! ${ }^{3}$ Hellingrath schickt mir, für Dich, beiliegende wissen- und gewissenhafte Hölderlin schrift, manchmal wohl etwas zäh und schrullig, aber im Ganzen höchst erfreulich.

Und nun Teuerster Meister, nur ein Wort zu deinem Geburtstag: dass ich alle tage weiss und fühle was dieser tag uns bedeutet und auferlegt! Mehr als dies immer neue bekenntnis zu dir und gelöbnis an dich, dich zu lieben und deiner liebe wert zu sein, Meister und Mittler, können wir dir nicht bringen. Nimm es hin mit deiner Güte und Nachsicht und fühl ein wenig von deinem eigenen sehnen und sehen erfüllt und bewährt in deinen getreuen, in der ehrfurcht und liebe deines Gundolf."

\section{Erläuterung}

Auf den Germanistikstudenten Friedrich Gundolf (1880-1931) hatte Karl Wolfskehl George im März 1899 aufmerksam gemacht. Gundolf wurde dann für viele Jahre zu Georges engstem Vertrauten und ist öffentlich wohl als erster akademisch von George kündender "Jünger" wahrgenommen worden. Er habilitierte sich 1911 in Heidelberg für Neuere deutsche Literaturgeschichte und bewegte sich, wie auch George, viel im Heidelberger Professorenumfeld. Enger Kontakt bestand so z.B. zum Nationalökonomen Eberhard Gothein und dessen Frau Marie Luise, den Eltern des hier als "SS" erwähnten Percy Gothein (1896-1944). George hatte den 14-Jährigen im September 1910 auf der Straße angesprochen, mit Einverständnis der Eltern dann Fotos von inm anfertigen lassen und ihn im Mai und September 1911 für je ein Wochenende nach Bingen eingeladen. Die Abkürzung "SS", "Sehr Süßer", spiegelt dabei die schon jenseits der Frage nach sexuellen Handlungen problematische erotische Aufladung, die die als potentielle "Zöglinge" angesehenen Jugendlichen im System um George ab dieser Zeit erfuhren. Georges Gedichtband "Der Siebente Ring" (1907), aus dem Gundolf beim Besuch Gotheins las, ist in dieser Hinsicht von symbolischer Tragweite: Er enthielt denjenigen zentralen Gedichtzyklus, in dem der Münchner Schüler Maximilian Kronberger (1888-1904), der zwischen seinem vierzehnten Lebensjahr und seinem frühen Tod zum Umfeld Georges gehört hatte, zur privatreligiös aufgeladenen Knabengottgestalt "Maximin" stilisiert wurde. Ebenfalls auf den Kontext des "Siebenten Rings" spielt Gundolf mit dem Hinweis auf Maulbronn an. In dem Band hatte George Orten wie Aachen, Bamberg, Hildesheim, München oder den "Gräbern in Speier" Gedichte gewidmet, die einen mythisch nationalkulturell aufgeladenen Raum deutscher Geschichte beschworen. Solche Bedeutung, legt Gundolf nahe, habe auch das ehemalige Zisterzienserkloster Maulbronn, dessen evangelische Klosterschule u. a. die Dichter Friedrich Hölderlin und Justinus Kerner besucht hatten. Hölderlin, dessen Hymne "Wie wenn am Feiertage..." 1910 durch das Verdienst Norbert von Hellingraths in einer Anthologie Georges erstveröffentlicht wurde, erhielt im "Kreis" um George die Aura des "grossen Seher[s] für sein volk" (George, Lobrede auf Hölderlin). In diesem Kontext ist auch die Nachricht, dass Hellingraths Dissertation „Pindarübertragungen von Hölderlin" erschienen sei, zu sehen. Im letzten Absatz feiert Gundolf dann Georges Geburtstag und kultiviert diesen zum großen Sinnereignis.

3) „Ernst der lange" meint Georges zeitweiligen Geliebten Ernst Morwitz (1887-1971), über dem der, gute Stern' der Autoren von Georges Zeitschrift "Blätter für die Kunst" gestanden habe, indem er nicht zum Militärdienst eingezogen worden war. 
Karl Bauer an Stefan George, 11.7.1918

Doppelblatt, 2 Seiten, mit Umschlag

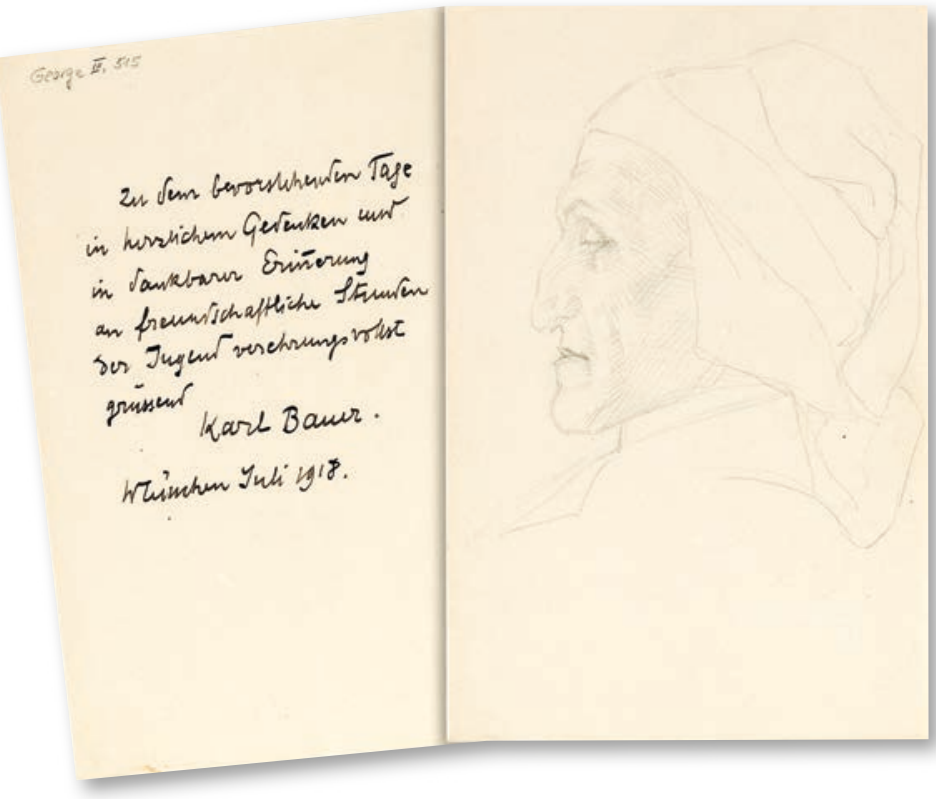

\section{Transkription}

„Zu dem bevorstehenden Tage in herzlichem Gedenken und in dankbarer Erinnerung an freundschaftliche Stunden der Jugend verehrungsvollst grüssend

Karl Bauer. München Juli 1918.“

\section{Erläuterung}

George lernte den gleichaltrigen, an der Kunstakademie in Stuttgart ausgebildeten Maler und Zeichner Karl Bauer (1868-1942) bereits 1891 in München kennen. Bauer, der ab Mitte der 1890er Jahre begonnen hatte, sich auf Porträtlithographien berühmter Persönlichkeiten zu spezialisieren, war von Georges Physiognomie fasziniert. Bis 1903 schuf er rund 30 Porträts, die George in meist herrscherlichem Gestus zeigen. Das bekannteste ist wohl seine Lithographie "Dichterbildnis mit Colleoni“ (1901), auf der George im Profil vor dem Venezianischen Reiterstandbild des Bartolomeo Colleoni zu sehen ist. Auch andere Schriftsteller wie Goethe, Schiller, Hauptmann oder Wedekind malte oder zeichnete Bauer in heroisierendem Stil. Im nationalsozialistischen Deutschland wurde er später dann für seine Darstellungen von Hitler und Goebbels ausgezeichnet.

Nach 1900 stand Bauer nur mehr sporadisch in Kontakt mit George. Ihm erging es hier wie fast allen Künstlerfreunden aus Georges Anfangsjahren, von denen dieser sich nach der Jahrhundertwende entfremdete. Mit der zum 50. Geburtstag verschick- ten Dantezeichnung, deren Mund- und Kinnpartie frappierend an Georges Profil erinnert, schlägt Bauer einen Bogen zurück zu den Jahren um 1900. George hatte damals an seinen Übertragungen aus der "Divina Comedia" gearbeitet und sich intensiv mit Dante auseinandergesetzt. Dieser präfigurierte für inn idealtypisch die Gestalt des die eigene Zeit kritisierenden, mit großer Strahlkraft ausgestatteten, über Jahrhunderte hinweg wirkmächtigen Dichter-Propheten. So stark war die Faszination, dass George im Fasching 1904 im Rahmen eines gemeinsam mit Wolfskehl, Maximilian Kronberger und anderen veranstalteten "Dichterumzugs" in der Kostümierung des Dante auftrat. Auf diese Selbstinszenierung als Dichter-Seher spielt die Dante-Zeichnung an.

\section{Reichspräsident Paul von Hindenburg an Stephan George, 11.7.1928}

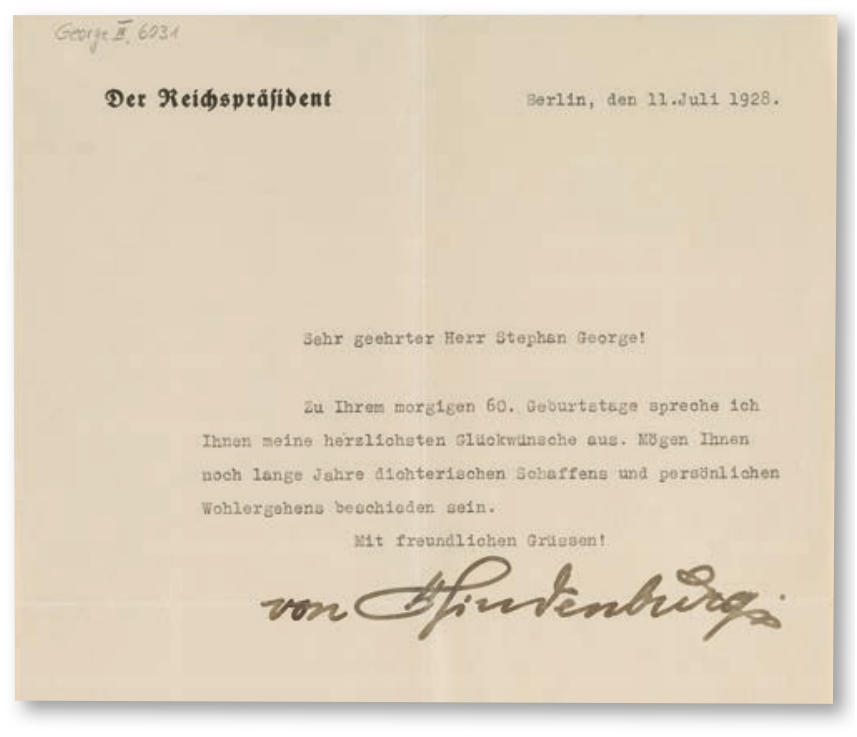

\section{Transkription}

„Berlin, den 11. Juli 1928

Sehr geehrter Herr Stephan George!

Zu Ihrem morgigen 60. Geburtstage spreche ich Ihnen meine herzlichsten Glückwünsche aus. Mögen Ihnen noch lange Jahre dichterischen Schaffens und persönlichen Wohlergehens beschieden sein. Mit freundlichen Grüssen! von Hindenburg."

\section{Erläuterung}

George hatte Paul von Hindenburg (1847-1934) 1917 in seinem Langgedicht "Der Krieg” im positiven Sinne als "schmucklosen Greis" bezeichnet, der das "reich" gerettet habe, und damit die von Hindenburg selbst mitbetriebene Mythologisie- 
rung seiner Person als vermeintlichem "Retter von Tannenberg" in einer Schlacht des Ersten Weltkriegs fortgeschrieben. Seit 1919 als Militär im Ruhestand, wurde Hindenburg im April 1925 als Nachfolger Friedrich Eberts 81jährig zum zweiten Reichspräsidenten der ersten deutschen Republik gewählt und gratulierte George in dieser Funktion als Staatsoberhaupt. Während die ebenfalls 1928 eingegangenen Glückwunschschreiben anderer hoher politischer und kultureller Repräsentanten wie etwa der Preußischen Akademie der Künste oder des Kultusministers Carl Heinrich Becker wie fast alle Glückwunschschreiben an George unbeantwortet blieben, reagierte George auf den Brief des Reichspräsidenten bereits am 15.7. mit einem persönlichen Dankesschreiben, das Hindenburg als „einzige sinnbildliche gestalt" lobt, die aus „den ungeheuren welt-wirren" der Zeit hervorrage.

\section{Claus Schenk Graf von Stauffenberg an Stefan George, 11.7.1931}

1 Doppelblatt, 4 Seiten

$$
\begin{aligned}
& 11 \text { Bad Kol6erg, 11.7.31. } \\
& \text { Main geliebrer Meiszer! } \\
& \text { Die mnigstan wanshe und alles shōne } \\
& \text { iu Doinem gebureszag: } \\
& \text { Dit hiesige kur hat glaubs in ganz gut } \\
& \text { getan. Inwit weit dor diromisnten sache } \\
& \text { abgefoleen we kams sivs nazunies ersi im } \\
& \text { lauf der Zest herausstellon. An mainem } \\
& \text { Magen ine mister posicises gatundon war } \\
& \text { den. Augerdings war die uncersuentung } \\
& \text { and rook wanis lit arreugend } \\
& \text { Dass of }
\end{aligned}
$$

\section{Transkription}

„Bad Kolberg, 11.7.31

Mein geliebter Meister!

Die innigsten wünsche und alles schöne zu Deinem geburtstag!
Die hiesige kur hat glaube ich ganz gut getan. Inwieweit der chronischen sache abgeholfen ist . kann sich natürlich erst im lauf der Zeit herausstellen. An meinem Magen ist nichts positives gefunden worden. Allerdings war die Untersuchung auch recht wenig überzeugend. Dass die Sache nervös sei ist die übliche und billige ausrede. Jedenfalls hat aber das sehr regelmässige und ruhige Leben hier auch dem Magen gut getan ausserdem habe ich das rauchen stark eingeschränkt; denn es ist kein wunder dass der handelsübliche misttabak für einen empfindlichen magen schädlich ist. Wetter war bis vor kurzem gut. Im übrigen war es hier aber unbeschreiblich langweilig. Durch die kur war man auch so behindert, dass man nichts vernünftiges anfangen konnte um die zeit tot zu schlagen. Ich habe jetzt für längere zeit ziemlich genug von der See und kann mir gut denken wie satt der Meister die Kieler landschaft nach einiger Zeit hatte. Mitte nächster woche fahre ich zurück nach Bbg[Bamberg]. wie sich der Sommer weiterhin für mich entwickeln wird wird sich erst dort herausstellen.

Hier kommen ständig wilde gerüchte über Berlin an. bisher war es ja immer unsinn. Trotzdem kann man sich des gefühls schwer erwehren · dass es dies jahr im Herbst oder Winter doch noch Ernst werden würde. Aber in solcher weise heute vorauszudenken ist ebenso undankbar als zwecklos. Das unvermeidliche wird doch kommen und dass es auf einige Jahre und einige menschenleben und schicksale mehr oder minder nicht ankommt glaube ich inzwischen gelernt zu haben. Küsse sehr den Frank. Es umarmt und küsst Dich Dein Claus."

\section{Erläuterung}

Claus Graf von Stauffenberg (1907-1944) war 15-jährig gemeinsam mit seinen älteren Zwillingsbrüdern Berthold und Alexander durch Maria Fehling, eine Freundin der Familie, und ihren damaligen Lebensgefährten Albrecht von Blumenthal bei George eingeführt worden. Der Zuwachs um die vermeintlichen Staufer-Nachfahren - Ernst Kantorowicz arbeitete damals bereits an seinem Buch über den Stauferkaiser Friedrich II. und bald bezeichnete sich Stauffenberg selbst in einem im November 1923 entstandenen Gedicht als „Erben“ der Staufer - wurde im Umfeld Georges begeistert aufgenommen. Nach dem Abitur schlug Stauffenberg im April 1926 die Offizierslaufbahn ein und trat dem 17. Reiterregiment in Bamberg bei. Diesem gehörte 
er auch im Sommer 1931 noch an, als er für einen Monat zur Behandlung allgemeiner Schwäche und eines Magenleidens im Ostseebad Kolberg war. Sein Glückwunschschreiben nach Königstein, wo George sich gerade zusammen mit Frank Mehnert, einem ehemaligen Schulkollegen Stauffenbergs aus Stuttgart und Vertrauten Georges, bei seiner Schwester Anna aufhielt, thematisiert in erster Linie die inm zu schaffen machende Ereignislosigkeit der Kur. Mehrfach schlägt Stauffenberg dabei Brücken zu George. So knüpft etwa die Erwähnung des schädlichen "Misttabaks" an die gemeinsame Gewohnheit des Rauchens an. Die Gleichförmigkeit der Seelandschaft erinnert an Georges Aufenthalte in Kiel bei Friedrich Wolters (gest. 1930) und im Hause der Familie Landmann. Und selbst im letzten Absatz, in dem Stauffenberg auf die Gefahr der Verschärfung der Wirtschaftskrise durch Reparationszahlungen, einen drohenden Bankenkrach sowie außenpolitische Spannungen mit Frankreich anspielt, greift er mit der Unterwerfung des Einzelschicksals unter die Idee des Ganzen, wie später Ernst H. Kantorowicz, einen der zwiespältigen Grundgedanken Georges auf.

\section{Propagandaminister Joseph Goebbels an Stefan George, 12.7.1933}

Telegramm

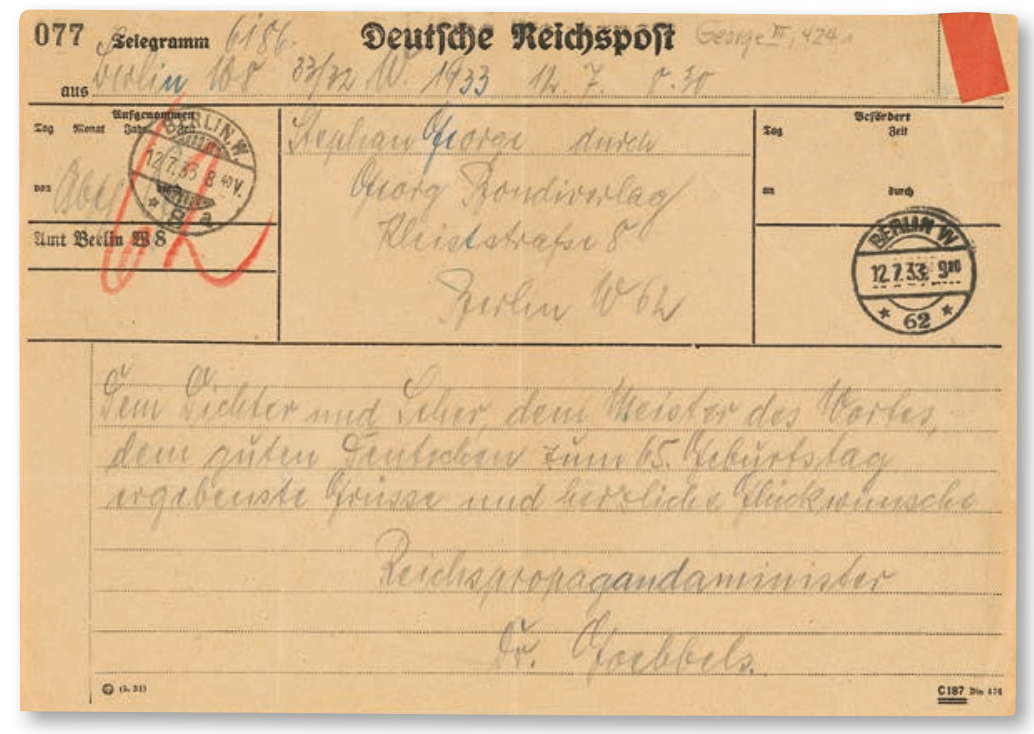

\section{Erläuterung}

Das offizielle Glückwunschschreiben des Reichsministers für Volksaufklärung und Propaganda Joseph Goebbels, der seine Aufgabe in der "geistigen Mobilmachung" Deutschlands sah, ruft nicht nur die im "Kreis" häufig benutzte Bezeichnung Georges als "Meister" auf und erklärt inn aus nationalsozialistischer Perspektive zum "guten Deutschen", sondern lobt inn bezeichnenderweise auch als "Seher". Der Text von Goebbels' Telegramm ging auch an die Presse. So gab die Deutsche Allgemeine Zeitung etwa bereits am Folgetag den Wortlaut wieder und malte mit Goebbels' Formulierungen die Verbindungslinie zwischen George und dem NS-Staat rückwärtig vereinnahmend aus: George erscheint dort als „einer der entscheidenden Führer der Nation und zu der Nation".

Dem Versuch der offiziellen Einbindung in die nationalsozialistische Kulturarbeit war George auf uneindeutige Weise aus dem Weg gegangen, indem er eine Anfrage aus dem Mai 1933, die Ehrenmitgliedschaft oder Präsidentschaft der Preußischen Akademie der Künste zu übernehmen oder einen Ehrensold des Staates zu empfangen, mit den Worten beantworten ließ: „irgendwelchen posten. auch ehrenhalber · der sogenannten akademie kann ich nicht annehmen ebensowenig einen sold. dass diese akademie jezt unter nationalem zeichen steht ist nur zu begrüssen und kann vielleicht später zu günstigen ergebnissen führen - ich habe seit fast einem halben jahrhundert deutsche dichtung und deutschen geist verwaltet ohne akademie · ja hätte es eine gegeben wahrscheinlich gegen sie. Anders verhält es sich mit dem positiven [...]: die ahnherrschaft der neuen nationalen bewegung leugne ich durchaus nicht ab und schiebe auch meine geistige mithilfewirkung nicht beiseite. Was ich dafür tun konnte habe ich getan - die jugend die sich heut um mich schart ist mit mir gleicher meinung.." (Entwurf der Antwort George/Mehnert an Morwitz, 10.5.1933, StGA)

\section{Transkription}

"Dem Dichter und Seher, dem Meister des Wortes, dem guten Deutschen zum 65. Geburtstag ergebenste Grüsse und herzliche Glückwünsche Reichspropagandaminister Dr. Goebbels." 


\section{Ernst Kantorowicz an Stefan George,} 10.7.1933

1 Blatt, 2 Seiten
George - . 66*2

Frankfure $\cdot 10$. VII. 33 .

\begin{abstract}
Geliebter Meiscer. Meine zeilen werden so fürcijos

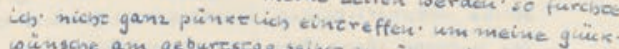

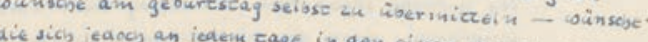

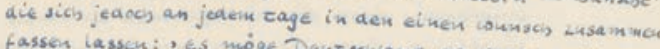
fassen iasscu: , es möge Deurscijana so deralen. coic es sies der Meiseer creniume sae.t. Und wenu das beutige

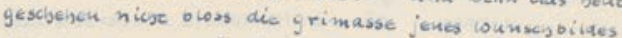

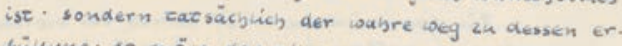
fühung. so möyc das alles zum guzen ausscisiagen und aann ist es gieidaghieig. ob aer cinzene auf he sem lokg mieschreizen kanh-vieimeinr: darf - oder

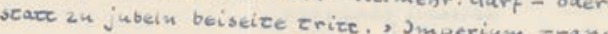
scendat hominem, erkiäree Friearicy I. und ies wäre der letzee der bier wiaerspricbe. versechen einem die taden alen zugang zum, reich. - una ais, jucle oder farbiger. wie die nene worz soppel lauzec. isz man von aem alein rassiscy fundierzen szaaz nocwenaig ausgescicossen - so wira man den amor faci aufbringen mussce una ism gomäss die enzscp hïese fassen. Indessen crbieit ich den beiliegenaen brief ron C. 4.K. Jch sciricb darauf hin an Berenam. Sprach aucis mit Saliu. der gerade bier war una mich besuchet iber die angeieger beit mic aem erfolg a abs jeder von beiaen - alkin oaker durch saimmining - eine getoisse
\end{abstract}

\section{Transkription}

„Geliebter Meister · meine zeilen werden · so fürchte ich · nicht ganz pünktlich eintreffen · um meine glückwünsche am geburtstag selbst zu übermitteln - wünsche · die sich jedoch an jedem tage in den einen wunsch zusammenfassen lassen: ıes möge Deutschland so werden - wie es sich der Meister erträumt hat!ı Und wenn das heutige geschehen nicht bloss die grimasse jenes wunschbildes ist . sondern tatsächlich der wahre weg zu dessen erfüllung · so möge das alles zum guten ausschlagen - und dann ist es gleichgültig · ob der einzelne auf diesem weg mitschreiten kann - vielmehr: darf - oder statt zu jubeln beiseite tritt. ıImperium transscendat hominemı erklärte Friedrich II. und ich wäre der letzte · der hier widerspräche. Verstellen einem die faten den zugang zum ıreich - und als sjude oder farbiger · wie die neue wortkoppel lautet - ist man von dem allein rassisch fundierten staat notwendig ausgeschlossen - so wird man den amor fati aufbringen müssen und ihm gemäss die entschlüsse fassen. - $[\ldots]^{4}$

Für den m'lichen brief danke ich vielmals und gebe die hoffnung nicht auf $\cdot$ d.M. im laufe des sommers noch sehen zu dürfen. Ich bleibe mit den allerbesten wünschen wie stets in treuer liebe und herzlichstem gedenken: Ernst."

\section{Erläuterung}

Ernst H. Kantorowicz (1895-1963) gibt im Glückwunschschreiben der paradoxen Hoffnung Ausdruck, dass der NS-Staat entgegen seiner eigenen Überzeugung der Beginn der Realisierung von Georges Phantasie eines neuen und besseren Deutschen Reichs sein möge. Falls dies so komme, werde er seinen als Jude bedingten ,schicksalhaften' Ausschluss aus diesem Reich akzeptieren. 1922 promoviert, verfasste der Historiker Kantorowicz in den Folgejahren in engem Austausch mit George seine Studie über den Stauferkaiser „Friedrich II". 1927 in Georges Buchreihe „Werke der Wissenschaft aus dem Kreise der Blätter für die Kunst" veröffentlicht, nahm das Buch historiographisch Teil an der Kultivierung der vormodernen Reichsidee, der sich George in seinen Gedichten nach 1900 immer stärker verschrieben hatte. Der zitierte Staufer-Satz, dass das ideelle „Reich den einzelnen Menschen überschreitet", markiert dabei den ungeheuerlichen Zwiespalt Kantorowicz' im Umgang mit George wie mit den neuen politischen Bedingungen nach dem Ende der Weimarer Republik: Der nationalkonservative Jude Kantorowicz war nach der Machtergreifung der Nationalsozialisten, die von einigen Georgeanern und nicht zuletzt seinem eigenen vormaligen Lebensgefährten Woldemar Graf Uxkull-Gyllenband als Staatswerdung von Georges Ideen begrüßt wurde, unmittelbar bedroht. Entgegen der im Brief nietzscheanisch als "amor fati“ gefassten Schicksalsergebenheit trat Kantorowicz im November 1933 mit einer Vorlesung über „Das Geheime Deutschland“ gegen die Identifizierung des völkischen NS-Staats mit Georges elitistischem ,Reichsideal' an: Angesichts der Disparatheit beider Reiche hatte er sich für mutige Konfrontation entschieden. Bald darauf setzten Schikanen der NS-Studentenschaft ein, und Kantorowicz gab seine Lehrtätigkeit an der Universität Frankfurt auf. Knapp vor einer drohenden Verhaftung emigrierte er 1938 über England in die USA.

\section{Maik Bozza / Birgit Wägenbaur}

4) Die in der Transkription gekürzte Passage handelt von einem Bittschreiben Carl August Kleins (1867-1952) und einer Geldsammelaktion für ihn im George-Umfeld. Von 1892 an und nominell bis zum Einstellen der Zeitschrift 1919 hatte Klein für George die Redaktion der Zeitschrift „Blätter für die Kunst" geführt. Schon vor 1900 in finanziellen Schwierigkeiten und bald kaum noch mit George verbunden, richtete er später immer wieder finanzielle Bittgesuche an George und sein Umfeld. 